\title{
Linear prediction studies for the solar wind and Saturn kilometric radiation
}

\author{
U. Taubenschuss ${ }^{1}$, H. O. Rucker ${ }^{1}$, W. S. Kurth ${ }^{2}$, B. Cecconi ${ }^{2}$, P. Zarka ${ }^{3}$, M. K. Dougherty ${ }^{4}$, and J. T. Steinberg ${ }^{5}$ \\ ${ }^{1}$ Space Research Institute, Austrian Academy of Sciences, A-8042 Graz, Austria \\ ${ }^{2}$ Department of Physics and Astronomy, The University of Iowa, Iowa City, Iowa 52242, USA \\ ${ }^{3}$ Laboratoire d'Etudes Spatiales et d'Instrumentation en Astrophysique, Observatoire de Paris, 92195 Meudon, France \\ ${ }^{4}$ Blackett Laboratory, Imperial College of Science and Technology, London SW7 2BZ, UK \\ ${ }^{5}$ Los Alamos National Laboratory, Los Alamos, New Mexico 87545, USA
}

Received: 4 April 2006 - Revised: 8 September 2006 - Accepted: 13 October 2006 - Published: 22 November 2006

\begin{abstract}
The external control of Saturn kilometric radiation (SKR) by the solar wind has been investigated in the frame of the Linear Prediction Theory (LPT). The LPT establishes a linear filter function on the basis of correlations between input signals, i.e. time profiles for solar wind parameters, and output signals, i.e. time profiles for SKR intensity. Three different experiments onboard the Cassini spacecraft (RPWS, MAG and CAPS) yield appropriate data sets for compiling the various input and output signals. The time period investigated ranges from DOY 202 to 326, 2004 and is only limited due to limited availability of CAPS plasma data for the solar wind. During this time Cassini was positioned mainly on the morning side on its orbit around Saturn at low southern latitudes. Four basic solar wind quantities have been found to exert a clear influence on the SKR intensity profile. These quantities are: the solar wind bulk velocity, the solar wind ram pressure, the magnetic field strength of the interplanetary magnetic field (IMF) and the y-component of the IMF. All four inputs exhibit nearly the same level of efficiency for the linear prediction indicating that all four inputs are possible drivers for triggering SKR. Furthermore, they act at completely different lag times ranging from $\sim 13 \mathrm{~h}$ for the ram pressure to $\sim 52 \mathrm{~h}$ for the bulk velocity. The lag time for the magnetic field strength is usually beyond $\sim 40 \mathrm{~h}$ and the lag time for the y-component of the magnetic field is located around $30 \mathrm{~h}$. Considering that all four solar wind quantities are interrelated in a corotating interaction region, only the influence of the ram pressure seems to be of reasonable relevance. An increase in ram pressure causes a substantial compression of Saturn's magnetosphere leading to tail collapse, injection of hot plasma from the tail into the outer magnetosphere and finally to an intensification of auroral dynamics and SKR emission. So, after the onset of magnetospheric
\end{abstract}

Correspondence to: U. Taubenschuss

(ulrich.taubenschuss@oeaw.ac.at) compression at least $\sim 1.2$ rotations of the planet elapse until intensified SKR emission is visible in a Cassini-RPWS dynamic spectrum.

Keywords. Magnetospheric physics (Solar windmagnetosphere interactions) - Radio science (Magnetosphere physics; Radio astronomy)

\section{Introduction}

Before the launch of the Cassini spacecraft, the knowledge of Saturn's radio emitting properties was based on data gained by the Voyager 1 and 2 missions (Warwick et al., 1981, 1982) and by the Ulysses spacecraft (Lecacheux et al., 1997).

The Saturn kilometric radiation (SKR) was detected for the first time when Voyager 1 was approaching Saturn in 1980 (Kaiser et al., 1980). This nonthermal radio emission usually occurs in the frequency range $3 \mathrm{kHz}-1.2 \mathrm{MHz}$ with a broad peak in flux density between $100-400 \mathrm{kHz}$ (Kaiser et al., 1984). The maximum flux density is at $\sim 3 \times 10^{-19} \mathrm{Wm}^{-2} \mathrm{~Hz}^{-1}$ normalized to a distance of $1 \mathrm{AU}$ to the planet. The spectrum typically looks bursty including also arc-like structures and bands and, according to recent Cassini observations, also fine structures if analyzed with time resolutions in the millisecond regime (Kurth et al., $2005 \mathrm{~b}$ ). Two components of opposite senses of nearly $100 \%$ circular polarization have been identified which propagate as $\mathrm{X}$-mode waves. Radiation coming from the northern hemisphere is right-handed polarized (RH) and radiation coming from the southern hemisphere is left-handed polarized (LH). Similar to radio wave phenomena observed at Earth and Jupiter, the Cyclotron Maser Instability (CMI) is an appropriate model for explaining the generation of SKR, too $(\mathrm{Wu}$ and Lee, 1979). The CMI theory describes the generation

Published by Copernicus GmbH on behalf of the European Geosciences Union. 
of planetary radio waves on the basis of a kinetic instability driven by a loss cone distribution for electron velocities.

The source regions of SKR are located in high magnetic northern and southern latitudes along auroral magnetic field lines. They are centered with respect to 13:00 LT near the noon meridian of Saturn above $80^{\circ}$ latitude. On the morning side between 08:00-09:00 LT possible source regions range down to $60^{\circ}$ latitude and on the evening side at 19:00 LT sources down to $75^{\circ}$ are possible (Galopeau et al., 1995). UV observations of bright aurora phenomena performed with the Hubble Space Telescope (HST) confirm the presence of energetic auroral electrons in the morning-to-noon sector (Trauger et al., 1998). Furthermore, HST observations show a nearly continuous UV auroral oval around the magnetic pole (Clarke et al., 2005) justifying the existence of SKR emission emitted from the nightside of Saturn as reported recently by Farrell et al. (2005).

Correlation studies between variations of the solar wind and SKR emission were published by Desch (1982) for the first time. He found clear correlations between the solar wind ram pressure $\rho v^{2}$ (mass density $\rho$, bulk velocity $v$ ) and SKR intensity in Voyager 1 and 2 data sets. A further indication of the strong dependence of SKR activity on the solar wind was found by Desch (1983). He detected a total disappearance of SKR during times when Saturn was moving through distant filaments of Jupiter's magnetotail and was thus shielded from the solar wind. Later on, Desch and Rucker $(1983,1985)$ improved those correlation studies using the superposed epoch method and a compilation of various solar wind quantities. They came to the conclusion that besides the ram pressure the solar wind momentum $(\rho v)$ and the kinetic energy $\left(\rho v^{3}\right)$ are also significant drivers for triggering SKR. The highest correlation coefficients have been found at zero lag time with a time resolution of $10.66 \mathrm{~h}$, i.e. data have been integrated over one full rotation period of Saturn. The interplanetary magnetic field (IMF) and its components revealed no clear correlation with SKR which may be due to the fact that solar wind measurements were performed up to $1.6 \mathrm{AU}$ ahead of Saturn and had to be ballistically propagated to the point of the planet thereby ignoring hydrodynamic interactions of high- and low-speed streams inside the solar wind.

The solar wind exerts its external control not only on SKR generation but also on Saturn's aurorae as investigated recently by Clarke et al. (2005) and Crary et al. (2005). New Cassini data in combination with ground-based and HST observations related Saturn's radio emission phenomena to detailed auroral structures (Kurth et al., 2005a).

The present paper re-analyses the external control of SKR by the solar wind with the application of the Linear Prediction Theory, outlined in Sect. 2. The data used as input and output time series are described in Sect. 3. The results are presented in Sect. 4 and the final Sect. 5 comprises the discussion and conclusion.

\section{Basics of the Linear Prediction Theory}

The Linear Prediction Theory (LPT) was developed by the American mathematician N. Wiener (Wiener, 1949), at first for continuous time series, and later on adapted by Levinson (1949) to discrete time series. The main aim of the LPT is to predict a function $Y$, called output signal, by convolving a filter function $f$ with another function $X$, called the input signal. For the discrete case the convolution equation looks like the following:

$Y_{t}=\sum_{s=-m}^{M-1} f_{s} X_{t-s}$.

A certain value $Y_{t}$ of the output is calculated by multiplying the filter coefficients $f_{s}$ with a sample of input data $X_{t-s}$ and then summing these weighted input data. If $X_{t}$ and $Y_{t}$ are time series representations then index $t$ indicates a certain time and index $s$ indicates the temporal shift or lag. A summation using only positive $s$-indices $(s=0, \ldots, M-1)$ is called the causal part of the filtering because only input data from the past are considered. Negative $s$-indices $(s=-m, \ldots,-1)$ contribute to the acausal part of the filtering which causes a physical conflict because it calculates output data taking input data from the future, i.e. an effect would precede the cause. Nevertheless, it could become important to calculate the acausal part too, especially for explaining strong filter reactions around lag position zero or to test the significance of a causal relationship.

The LPT works exclusively with a non-recursive filter as outlined in Eq. (1) and furthermore, the filter has to be linear and time invariant. Additionally, the time series used must be sampled from a stochastic process and the time series must be stationary.

The Linear Prediction Theory is usually applied on the condition that the input signal $X_{t}$ and the measured output signal $Z_{t}$ are known by means of corresponding measurements and that the filter coefficients $f_{s}$ have to be calculated. The measured output is named $Z_{t}$ in order to distinguish it from the calculated output $Y_{t}$. The filter $f_{s}$ must be constituted such that $Y_{t}$ becomes the best possible representation of $Z_{t}$ as far as their linear relationship is concerned. So, the mean-square-error between $Z_{t}$ and $Y_{t}$ has to be minimized. Stressing the convolution equation in combination with the statistical criteria of least squares fitting makes a calculation of the filter coefficients possible. The relationships between input, outputs and filter are sketched in Fig. 1.

The procedure for calculating the filter coefficients of a single-channel filter is presented in the Appendix. As a last step, the filter coefficients together with the input $X_{t}$ have to be inserted into the convolution equation (1) to get the calculated output signal $Y_{t}$. 
A parameter for quantifying the degree of fit between the calculated output $Y_{t}$ and the measured output $Z_{t}$, i.e. the performance of the linear prediction, is the efficiency defined as

$e f f=\left(1-\frac{\sigma_{r}^{2}}{\sigma_{z}^{2}}\right) \times 100 \quad[\%]$.

$\sigma_{z}^{2}$ is the variance of $Z_{t}$ and $\sigma_{r}^{2}$ is the variance of the residual time series $\left(Z_{t}-Y_{t}\right)$. An efficiency of $+100 \%$ means that all variations of the measured output $Z_{t}$ are reproduced by the variations of the calculated output $Y_{t}$. On the other hand, a negative value for eff indicates that $Y_{t}$ does not reproduce the variations of $Z_{t}$ and hence the prediction of the output $Z_{t}$ by the input $X_{t}$ is wrong. Moreover, the efficiency can be investigated as a function of the temporal shift parameter $s$. Therefore, the output $Y_{t}$ has to be calculated with a successively increasing maximum shift. The filtering process is said to be completed if an additional extension of the filter does not raise the efficiency any more.

Finally, some further important remarks on the LPT are given. It is important that the signals which are applied to the LPT analysis contain the same number of discrete data points and exhibit the same time resolution. Thus, the signals must comprise the same time period. Furthermore, the maximum allowed number of discrete shifts, i.e. $(M+m)$, is limited to $15 \%$ of the overall number $N$ of data points (Taubenheim, 1969; Schönwiese, 1985).

An exact representation of the measured output $Z_{t}$ by the calculated output $Y_{t}$ is only possible if the summation in Eq. (1) is performed from negative infinity to positive infinity and if the relation is strictly linear and time invariant. These conditions can hardly be fulfilled by real measured signals and one has to accept the fact that only a certain level of efficiency lower than $100 \%$ will be achieved. So, the disadvantage of the LPT is the fact that it ignores relations having higher moments of the investigated process. Moreover, a filter is only valid for the time period selected for the calculations. On the other hand, it simplifies the analysis as a first approximation by assuming a linear system. Even if the relation between the input and output is not strictly linear, the Linear Prediction Theory will calculate the best possible linear relation.

The scenario described above using one measured input and one measured output is called single-channel system. As an extension, a so-called multi-channel system operates with more than one input and output. If the LPT is carried out in multi-channel mode, all signals will be processed collectively, thereby not ignoring relations among the individual signals. The computation of the filter coefficients follows the same concept as outlined in the appendix for the single-channel system except that cross-correlation and auto-correlation coefficients have to be arranged in tensors of higher dimension.

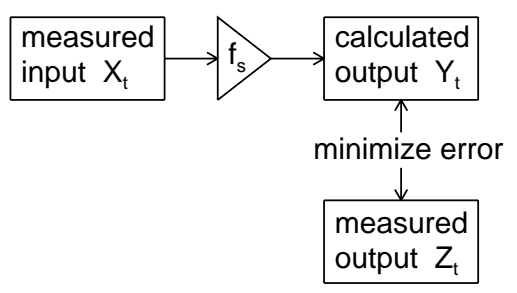

Fig. 1. Schematics of the concepts of the Linear Prediction Theory. The measured input $X_{t}$ is filtered (filter function $f_{s}$ ) yielding a calculated output $Y_{t}$ which is then compared to the actually measured output $Z_{t}$. The error power between $Y_{t}$ and $Z_{t}$ shall be minimized.

\section{Preparation of input and output signals}

The Linear Prediction Theory was used to investigate the influence of the solar wind on SKR intensity. Profiles for various solar wind quantities serve as input signals and SKR integrated intensity-time profiles serve as output signals. Data obtained by three different experiments onboard the Cassini spacecraft have been used to construct input and output signals feeding the LPT algorithm. These three experiments are the Radio and Plasma Wave Science experiment (RPWS) (Gurnett et al., 2004), the Dual Technique Magnetometer (MAG) (Dougherty et al., 2004) and the Cassini Plasma Spectrometer (CAPS) (Young et al., 2004).

SKR intensity profiles are established using data from the RPWS experiment. One important point is that these profiles use only SKR emissions. SKR emissions can be clearly identified in a dynamic spectrum regarding the frequency range and the polarization characteristics. SKR usually occurs in the frequency range $3-1200 \mathrm{kHz}$ and exhibits a high degree of circular polarization (nearly 100\%). The polarization of measured radio waves was determined by applying the Direction-Finding algorithm for a 3-axis stabilized spacecraft using a direct inversion (Cecconi and Zarka, 2005a). The Direction-Finding analysis yields not only the full polarization information of the received radio waves, i.e. the Stokes parameters, but also eliminates the influence of intensity variations which are due to a changing orientation of the antenna system with regard to the direction of the radio source. Figure 2 demonstrates the results of the DirectionFinding computations as far as the polarization is concerned. As can be seen, the dominant polarization sense of SKR is left-handed (LH) which corresponds well to the fact that Cassini was positioned at low southern latitudes $\left(l a t \approx-17^{\circ}\right.$, $\left.r \approx 150 \mathrm{R}_{\mathrm{S}}\left(1 \mathrm{R}_{\mathrm{s}}=60268 \mathrm{~km}\right), \mathrm{LT} \approx 5.6 \mathrm{~h}\right)$ during the considered time period DOY 232.5-234.0, 2004. Thus SKR emission from source regions mainly in the southern hemisphere was beamed towards Cassini.

Solar wind parameters used as input signals are measured by the CAPS and MAG experiments. The latter yields measurements of the interplanetary magnetic field in a defined 


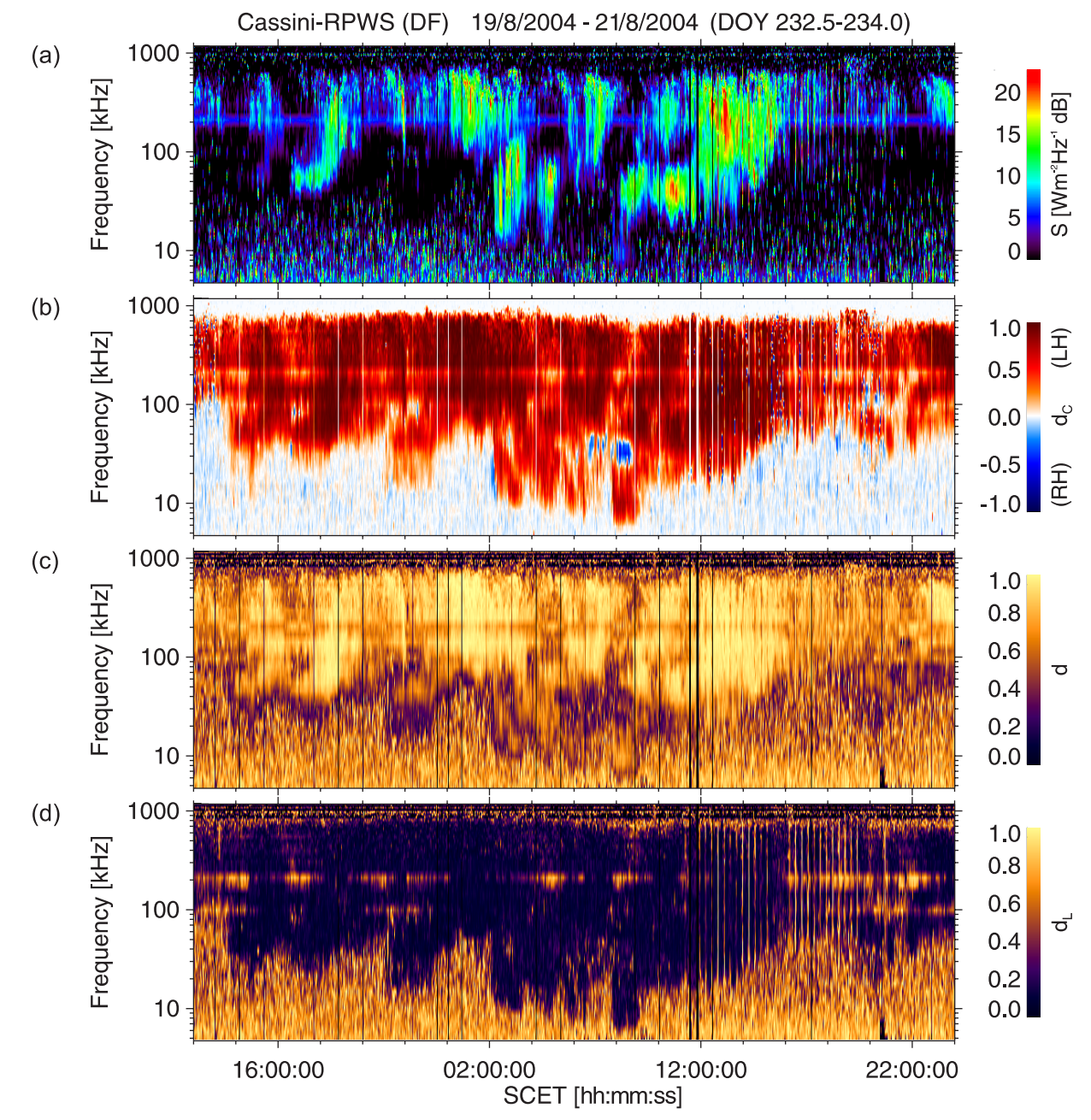

Fig. 2. Cassini-RPWS dynamic spectra for (a) the Stokes parameter S (= total intensity), (b) the degree of circular polarization $d_{c}$, (c) the degree of polarization $d$ and (d) the degree of linear polarization $d_{L}$ as a result of the Direction-Finding computations for the time period DOY 232.5-234.0, 2004.

coordinate frame. The CAPS-experiment provides plasma parameters like the solar wind bulk velocity, proton density and proton temperature.

Magnetic field and plasma measurements can be combined into several other quantities describing the physical state of the solar wind and yielding additional input signals for the LPT computations.

The solar wind quantities investigated in the frame of the LPT are:

- the solar wind bulk velocity $v$, the ram pressure $\rho v^{2}$ and the proton temperature $T_{p}$ (Desch and Rucker, 1983)

- the parameters $B^{2} v$ (dynamo energy flux) and $B v^{2}$ (correlates well with AKR at Earth; Gallagher and D'Angelo, 1981)

- the parameter $B_{z} v^{2}$ describing the erosion of planetary magnetic field lines
- the reconnection voltage $\Phi=v B_{T} L_{0} \cos ^{4}(\theta / 2)$ describing the voltage along the reconnection line for dayside magnetopause reconnection between Saturn's magnetic field and the IMF (Jackman et al., 2004). The IMF magnetic field vector and the magnetic dipole axis of Saturn have to be rotated into a coordinate frame whose $y z$-plane is oriented perpendicular to the direction of $\boldsymbol{v}$. The magnetic axis is assumed to be coaligned (within $\sim 1^{\circ}$ ) with Saturn's spin axis. Then the angle $\theta$ is a polar angle for $B_{T}\left(B_{T}^{2}=B_{y}^{2}+B_{z}^{2}\right)$ counted from Saturn's magnetic North Pole. The quantity $v B_{T}$ refers to the motional electric field in the planet's rest frame $\left(\boldsymbol{v} \perp \boldsymbol{B}_{T}\right) . L_{0}$ is a factor for the dimension of the magnetospheric cross-section $\left(L_{0} \approx 10 \mathrm{R}_{\mathrm{s}} ; 1 \mathrm{R}_{\mathrm{s}}=60268 \mathrm{~km}\right)$. An integration of $\Phi$ over time yields the rate of open flux production. 
- the Akasofu-parameter $\varepsilon=B^{2} v L_{0}^{2} \cos ^{4}(\theta / 2) \quad$ (Akasofu, 1978) and the modified Akasofu-parameter $\varepsilon_{\text {mod }}=B_{T}^{2}(v / \rho)^{1 / 3} \cos ^{4}(\theta / 2)$ (Vasyliunas et al., 1982). The quantities $B_{T}, L_{0}$ and $\theta$ have the same meaning as discussed for the reconnection voltage.

- and the quantity $Q=\frac{\mu_{0}\left(v_{2}-v_{1}\right)^{2}}{B_{1}^{2}\left(1 / \rho_{1}+1 / \rho_{2}\right)}$ describing the probability that a Kelvin-Helmholtz instability occurs at the dayside magnetopause initiating an increased flow of charged particles into the Saturn auroral regions (Galopeau et al., 1995) (index 1 refers to parameters in the magnetosheath just outside the magnetosphere and index 2 refers to parameters inside the magnetosphere of Saturn)

The solar wind momentum $(\rho v)$ and the kinetic energy $\left(\rho v^{3}\right)$ have not been investigated explicitly. The corresponding time profiles look very similar to the time profile of the ram pressure if centered around the mean and normalized to standard deviation units as performed during the LPT computations. Variations of the density seem to be definitely more dominant than variations of the bulk velocity.

\section{Results}

The total time period for which data are available is limited to the period DOY 202-326, 2004 due to limited availability of CAPS plasma data because the CAPS instrument was not always pointed properly to view the solar wind ions. During DOY 297-311, 2004 Cassini was positioned inside Saturn's magnetosphere and no measurements for the solar wind could have been performed. Large data gaps ( $>1$ day) in the CAPS data occurring after DOY 260 cannot be represented satisfactorily by interpolated values and thus the period DOY 260-326 had to be rejected. In the following, the results of the LPT computations found for the time period DOY 224-240, 2004 will be presented showing an evident triggering effect of the solar wind on SKR. According to the statistical criteria of the LPT, a period of 16 days allows for performing a maximum shift in time of $57.6 \mathrm{~h}(15 \%)$ which corresponds to 5.4 rotations of Saturn.

The period DOY 224-240, 2004 was selected because it is centered around two clear peaks in the SKR intensity profile occurring on DOY 233.0 and DOY 233.5 as can be seen in Fig. 3b. The intensity profile was established by integrating SKR intensities present in the RPWS dynamic spectrum (shown in Fig. 3a), averaging the profile to a time resolution of $20 \mathrm{~min}$ and normalizing intensities to a distance of $1 \mathrm{AU}$ to Saturn. Rapid fluctuations are removed from the profiles by applying a low-pass $\cos ^{2}$-filter with a length of $5 \mathrm{~h}$ in time domain. The time period displayed in the previous Fig. 2 was selected such that this region of intensified SKR emission is provided.

In Fig. 3b many small $\perp$-symbols plotted directly below the SKR intensity profile indicate minima which are caused by the rotation of Saturn. Neighboring $\perp$-symbols are separated by $10.75 \mathrm{~h}$, i.e. the present radio rotation period for Saturn according to Gurnett et al. (2005). In Fig. 3c profiles for the solar wind ram pressure $P_{\text {ram }}$ (solid line) and the solar wind bulk velocity $v_{\text {bulk }}$ (dotted line) are displayed. In Fig. 3d the profiles for the magnetic field strength $B$ (solid line) and the $y$-component $B_{y}$ (dotted line) of the IMF in KSM-coordinates are shown. KSM denotes "Kronocentric Solar Magnetospheric" and is similar to the GSM system used for Earth. The $X$-axis points from Saturn to the sun, i.e. it is aligned with the local time 12:00 direction. The $Y$-axis is perpendicular to the plane stretched by the $X$-axis and the rotation axis $\Omega$ of Saturn $(\boldsymbol{Y}=\boldsymbol{\Omega} \times \boldsymbol{X})$. The $Z$-axis completes the right-handed coordinate system. Saturn's rotation axis and magnetic dipole axis differ by $<1^{\circ}$, so the KSM coordinate system can also be related to Saturn's magnetic field. Finally, Fig. 3e presents the profile of the dayside magnetopause reconnection voltage $\Phi$. For the sake of clarity additional profiles for the other input signals listed above in Sect. 3 are not included.

A strong increase of both the bulk velocity (around DOY 231.5) and the ram pressure (peak at DOY 232.9) indicates the arrival of an interplanetary shock at Saturn. Shocks are generated by high-speed plasma flows escaping from coronal holes and overtaking slower moving solar wind plasma. The resulting compression of plasma is followed by several days of rarefaction during which the solar wind speed continuously falls. If such a characteristic pattern of the interplanetary medium is repeated for several successive rotations of the sun, it is called "corotating interaction region" (CIR) (Tsurutani et al., 1982; Gosling, 1996). Since the interplanetary magnetic field is frozen into the plasma $B$ is also enhanced in a compression region. The profile for the $B_{y}$-component reveals that the CIR includes a heliospheric current sheet (HCS)-crossing around DOY 232.5 when $B_{y}$ changes its sign from negative to positive. This corresponds to a change in the orientation of the tangential component of the IMF from a towards-configuration to an away-configuration with regard to the position of the sun. The reconnection voltage generally undulates below $100 \mathrm{kV}$ with a peak of $138 \mathrm{kV}$. During the period DOY 224-233, i.e. a few days before the strong intensification of SKR, low values for $\Phi$ of a few tens of $\mathrm{kV}$ are due to low values of $B_{T}$ and a mainly southward oriented IMF $\left(\theta>90^{\circ}\right)$. The rate of open flux production accumulated during these 9 days is about $11 \mathrm{GWb}$ (Gigaweber) which is less than the typical amount of open flux in Saturn's tail $(\sim 35 \mathrm{GWb})$ deduced from HST aurora observations (Jackman et al., 2004).

In the following, the efficiency functions for the various input signals, i.e. the efficiency as a function of the temporal shift between input and output, will be presented as the key result of the LPT computations (see Eq. 2). The efficiency reveals the characteristics of the linear filter found by the LPT algorithm. 


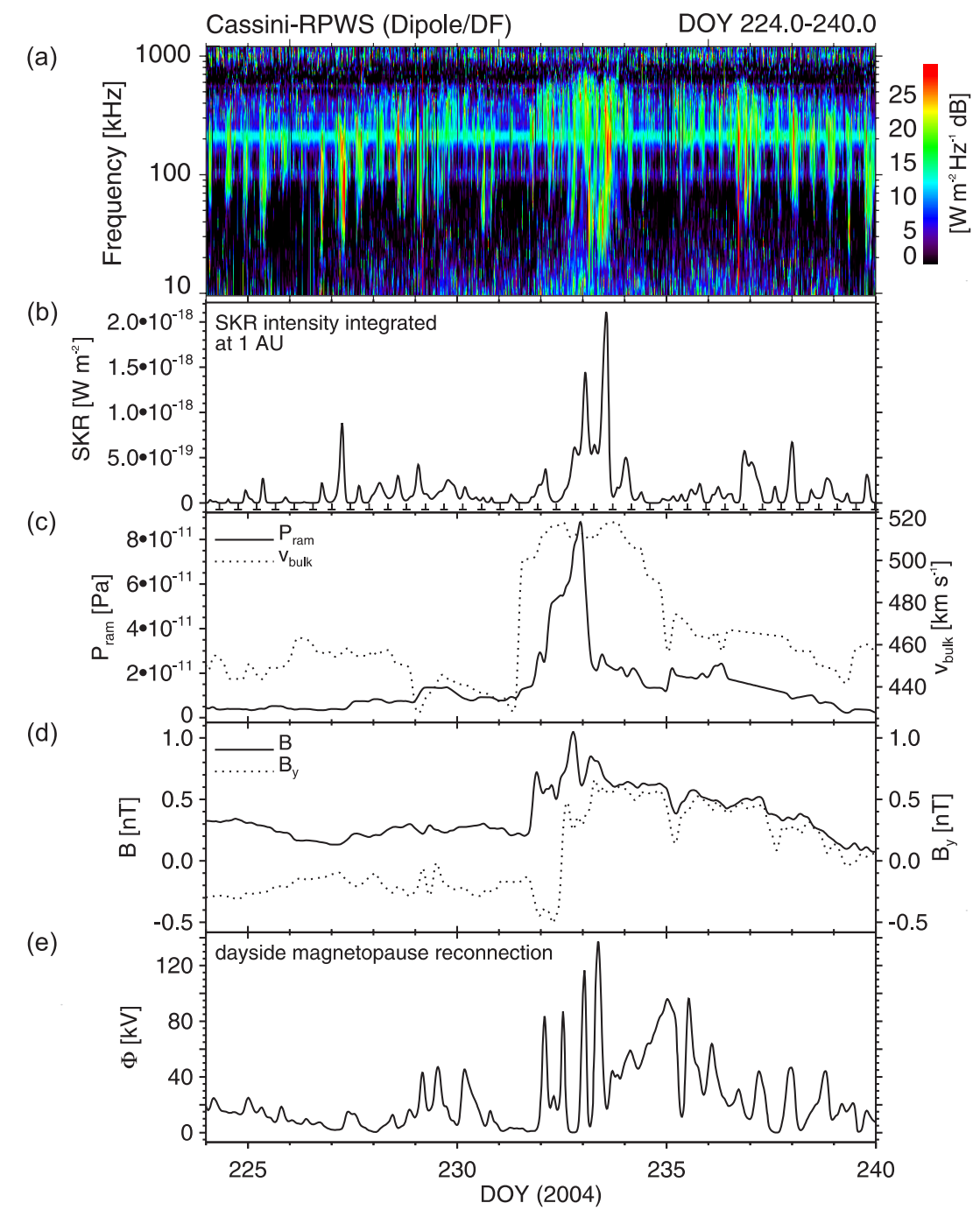

Fig. 3. (a) The RPWS dynamic spectrum, (b) the integrated SKR intensity profile, (c) profiles for the SW ram pressure (solid) and bulk velocity (dotted), (d) profiles for the interplanetary magnetic field strength (solid) and its y-component (dotted) in KSM-coordinates and (e) the profile of the reconnection voltage at the dayside magnetopause of Saturn during DOY 224-240, 2004.

First, it was found that using the time derivative of an input quantity or the absolute value of the input's time derivative yields a smoother efficiency function than using the original input. For illustration, Fig. 4 displays the efficiency functions for the ram pressure and $B_{y}$ in combination with the integrated SKR intensity as output signal, respectively. If the time derivative of the input is used, the input is flagged with an additional $\delta$-symbol. An efficiency function for the absolute time derivative of the input is flagged with $a b s(\delta)$.

The solid line in Fig. 4a belongs to the efficiency of the ram pressure without performing any derivative. Strong fluctuations at small lag times including also negative values are obvious. Negative values indicate that the prediction of SKR intensity by the ram pressure is wrong for these small lag times. Between $12-13 \mathrm{~h}$ lag time the efficiency function exhibits a significant increase. This implies that at this temporal shift the influence of variations from the input on variations of the output is a maximum and that this temporal shift may be interpreted as the temporal lag required for the input to trigger the output. Furthermore, the efficiency function levels off at a constant plateau level after $14 \mathrm{~h}$ lag time. This means that introducing larger shifts between input and output does not raise the efficiency anymore, so, the filtering between input and output is completed after a lag time of $14 \mathrm{~h}$. Figure $4 \mathrm{a}$ also includes efficiency functions for the time derivative (dotted line) and the absolute value of the time derivative (dashed line) of the ram pressure. One recognizes that the efficiency function for $\delta P_{\text {ram }}$ increases smoothly until the maximum 
(a)

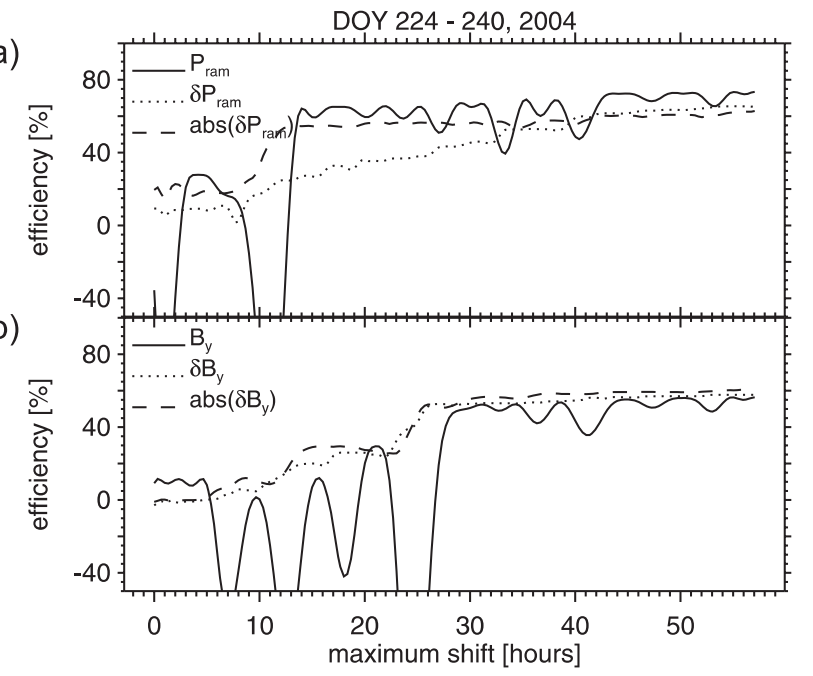

Fig. 4. Efficiency functions for (a) the ram pressure $P_{\text {ram }}$ and (b) the $B_{y}$-component of the IMF in combination with SKR intensity as output signal for the time period DOY 224-240, 2004. The respective time derivatives of the inputs are flagged with $\delta$ and the absolute time derivatives are flagged with $a b s(\delta)$.

temporal shift of $57.6 \mathrm{~h}$ is reached. No significant increase and no constant plateau level is visible indicating a bad linear prediction. For $a b s\left(\delta P_{\text {ram }}\right)$ the situation is different. The corresponding efficiency function shows a steep increase between $10-12 \mathrm{~h}$ lag time, i.e. $\sim 2 \mathrm{~h}$ earlier than for the normal input. Fluctuations in the efficiency function of $a b s\left(\delta P_{r a m}\right)$ are weaker than in $P_{\text {ram }}$ making a characteristic lag time easier to identify.

In Fig. $4 \mathrm{~b}$ the efficiency functions for $B_{y}$ and its derivatives are displayed. Again, $a b s\left(\delta B_{y}\right)$ (dashed line) reveals a smoother efficiency function than $B_{y}$ (solid line) and a constant plateau level is achieved at $\sim 26 \mathrm{~h}$ lag time. The efficiency function for $\delta B_{y}$ (dotted line) also suggests a completed filter after $26 \mathrm{~h}$. The efficiency function for the normal input quantity $B_{y}$ starts to level off about $2 \mathrm{~h}$ later, i.e. after $\sim 28 \mathrm{~h}$.

So, as demonstrated with $P_{r a m}$ and $B_{y}$ in Fig. 4, a better efficiency function is obtained by taking the time derivative or the absolute value of the time derivative of the respective input quantity. A good linear prediction is expressed by an efficiency function exhibiting a steep increase at a certain lag time and a subsequent constant plateau level. The higher the plateau level, the better the linear prediction. Plateau levels below $30 \%$ are classified as insignificant predictions.

Such insignificant or not well-defined predictions are obtained for the following input parameters: the solar wind proton temperature, the magnetic components $B_{x}$ and $B_{z}$, the modified Akasofu-parameter, the reconnection voltage and the Kelvin-Helmholtz instability quantity. The corresponding efficiency functions are displayed in Figs. 5a and 5b.

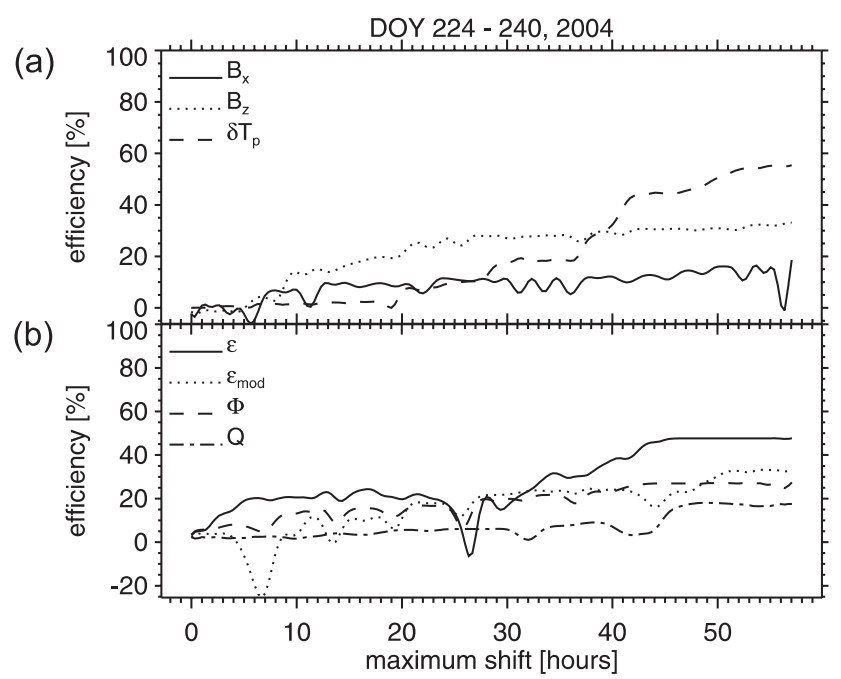

Fig. 5. In (a) the efficiency functions for the magnetic components $B_{x}$ and $B_{z}$ and the efficiency function for the time derivative of the SW proton temperature $\delta T_{p}$ are displayed. Part (b) shows the efficiency functions for the Akasofu-parameter $\varepsilon$, the modified Akasofu-parameter $\varepsilon_{m o d}$, the reconnection voltage $\Phi$ and the KHinstability quantity $Q$.

The efficiency functions for $B_{x}$ (solid line) and $B_{z}$ (dotted line) are settled at low levels and exhibit no significant increase. Taking the time derivatives or absolute time derivatives does not improve the result. For the proton temperature the time derivative $\delta T_{p}$ smoothes strong fluctuations in the efficiency function if compared to the original $T_{p}$, but a constant plateau level is not achieved (see dashed line in Fig. 5a). This does not exclude the possibility that a significant influence of $T_{p}$ on SKR may be found at larger lag times beyond $57.6 \mathrm{~h}$. The efficiency function for the KHinstability quantity $Q$ (dashed-dotted line in Fig. 5b) reveals a poor prediction with efficiencies below 20\%. Even taking the time derivative or absolute value of the time derivative of $Q$ does not improve the result. In comparison to the modified Akasofu-parameter $\varepsilon_{\text {mod }}$ (dotted line), the normal Akasofuparameter $\varepsilon$ (solid line) yields better results exhibiting a clear plateau level of $47 \%$ after $\sim 45 \mathrm{~h}$ lag time. The efficiency function for the reconnection voltage $\Phi$ (dashed line) shows a similar behavior as the efficiency function of $\varepsilon_{\text {mod }}$ because both quantities are dominated by variations of $B_{T}$ and the polar angle $\theta$.

Another characteristic found was that if the bulk velocity and the magnetic field are mixed, e.g. in $B^{2} v, B v^{2}$ or $B_{z} v^{2}$, then $v$ seems to be of minor influence. Consequently, the efficiencies of $B^{2} v, B v^{2}$ and $B_{z} v^{2}$ are nearly the same as for $B$ or $B_{z}$ alone. The same is true if $v$ is mixed with the density $\rho$. As mentioned at the end of Sect. 3 , the profiles for $\rho v, \rho v^{2}$ and $\rho v^{3}$ look very similar thus yielding similar efficiency functions. 


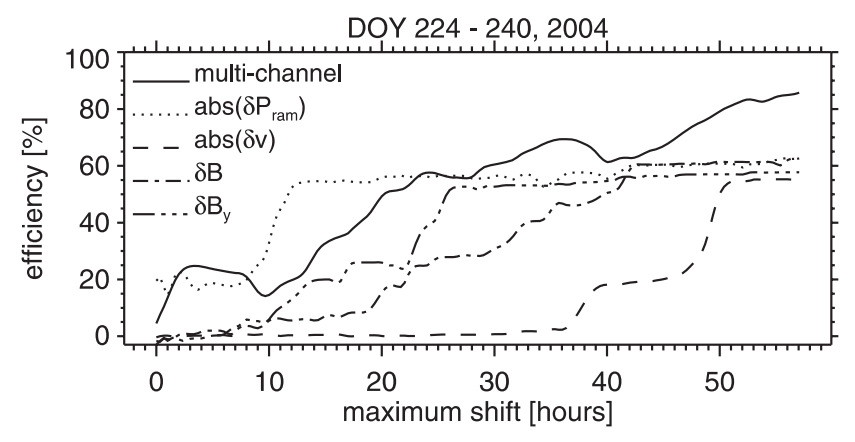

Fig. 6. The efficiency as a function of the temporal shift for the ram pressure $P_{\text {ram }}$ (dotted), the bulk velocity $v$ (dashed), the magnetic field strength $B$ (dash-dot) and the magnetic component $B_{y}$ (dashdot-dot-dot). The time derivative is indicated by $\delta$, the absolute value of the time derivative is indicated by $a b s(\delta)$. The solid line is produced if all 4 input signals are mixed in a multi-channel filter.

Finally, four characteristic input signals are left yielding the best results as far as the linear prediction of SKR is concerned. These four input quantities are the ram pressure $P_{\text {ram }}$, the bulk velocity $v$, the magnetic field strength $B$ and the $y$-component $B_{y}$ of the IMF given in KSM-coordinates. The respective efficiency functions for the time derivative or the absolute value of the time derivative are summarized in Fig. 6. Moreover, Fig. 6 includes the efficiency if all four input signals are processed together in a multi-channel filter (solid line).

It can clearly be seen that the efficiency functions for the absolute time derivative of $P_{r a m}$ and $v$ and for the time derivative of $B_{y}$ exhibit a significant increase in efficiency at certain temporal shifts. Together with $\delta B$ all four efficiency functions level off at a constant plateau level. The characteristic lag times are $\sim(12+1) \mathrm{h}$ for $P_{\text {ram }}, \sim(51+1) \mathrm{h}$ for $v$, $\sim(43+1)$ h for $B$ and $\sim(26+1)$ h for $B_{y}$. The additional lag time $(+1) \mathrm{h}$ indicates that a characteristic solar wind structure arrives about $1 \mathrm{~h}$ earlier at the dayside magnetopause than at Cassini. This is due to the position of Cassini on its orbit around Saturn and causes an additional acausal shift for the LPT computations which has to be removed. The position of the dayside magnetopause of Saturn was calculated on the basis of ram pressure data in combination with the magnetopause model of Slavin et al. (1985). During the time period DOY 224-240, 2004 Cassini was positioned mainly on the morning side around 06:00 LT. So, e.g. for the ram pressure, it can be concluded that the physical processes involved to transform an increase in ram pressure into an increase in SKR intensity require about $13 \mathrm{~h}$ as observed by the Cassini spacecraft.

As mentioned before, the efficiency functions for all four input quantities achieve a plateau level at 55\%-60\% efficiency, so, all four input quantities seem to trigger SKR to the same degree of probability.

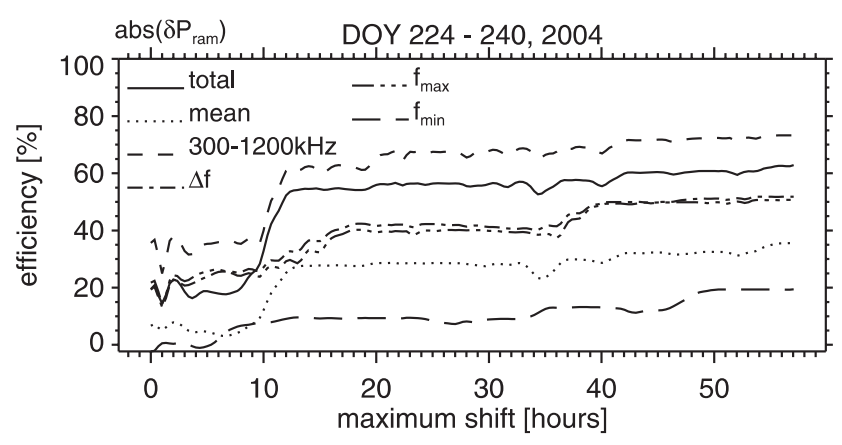

Fig. 7. Efficiency functions for the absolute time derivative of the ram pressure $a b s\left(\delta P_{\text {ram }}\right)$ using six different output signals: total SKR intensity integrated over the whole frequency range (solid line), mean intensity (dotted), intensity integrated from $300-1200 \mathrm{kHz}$ (short dashes), frequency bandwidth (dash-dot), upper SKR frequency limit (dash-dot-dot-dot) and lower SKR frequency limit (long dashes).

The solid line in Fig. 6 represents the efficiency function if all 4 inputs are mixed in a multi-channel filter. As can be seen, the efficiency function climbs up to very high values above $80 \%$ but it does not level off at a constant plateau. So, investigating the external control of SKR in the frame of a multi-channel filter does not seem to improve the result.

So far, the input signals have been transformed into their respective derivatives to enhance the quality of the linear prediction. SKR intensity integrated over the whole frequency range was used as output signal. The following five manipulations of the output signal have been tested to check if the results for the efficiency functions can be further enhanced by

- using a mean SKR intensity instead of the integrated total intensity across the whole frequency range

- skipping the lower frequency range by integrating intensities just from $300-1200 \mathrm{kHz}$

- using the SKR frequency bandwidth $\Delta f$ as output

- using the SKR maximum frequency $f_{\max }$ as output

- using the SKR minimum frequency $f_{\min }$ as output. The resulting efficiency functions for these five manipulations using $a b s\left(\delta P_{\text {ram }}\right)$ as input signal are displayed in Fig. 7. If compared to the total SKR intensity taken as input signal (solid line) an integration of intensities just from $300-1200 \mathrm{kHz}$ (short dashes) raises the efficiency function about $10 \%$ but fluctuations are a little bit stronger and the increase in efficiency around $11 \mathrm{~h}$ lag time is less distinct. Taking a mean intensity as output signal lowers the efficiency level significantly (dotted line). If the SKR frequency bandwidth (dash-dot) is used for output nearly the same result is obtained as taking the upper frequency limit (dash-dot-dotdot). The lower frequency limit shows the worst results with efficiencies $<20 \%$ (long dashes). 
These modifications of the output signal have also been executed using $v, B$ and $B_{y}$ as inputs. The respective transformations of the efficiency functions for the different outputs show the same behavior as for the ram pressure displayed in Fig. 7. Another test performed was using the time derivative or absolute value of the time derivative not only for the inputs but also for the output. No improvements for the linear filter are obtained if these derivatives are applied to the SKR intensity profile both in combination with plasma quantities and magnetic field quantities.

\section{Summary and discussion}

The Linear Prediction Theory has been applied to Cassini data to investigate the external control of SKR by the solar wind. The results can be summarized as follows:

- Taking the time derivative of an input quantity or the absolute value of its time derivative enhances the efficiency function significantly. Characteristic lag times become more distinct and fluctuations of plateau levels are suppressed enabling a better interpretation. It seems that variations of a solar wind input are more important than magnitude values for predicting the SKR intensity profile.

- Four basic input signals $\left(P_{\text {ram }}, v, B\right.$ and $\left.B_{y}\right)$ have been found to trigger SKR with nearly the same efficiency but at completely different lag times. These lag times and efficiency levels are: $P_{\text {ram }} \rightarrow(\sim 13 \mathrm{~h}, 55 \%), v \rightarrow$ $(\sim 52 \mathrm{~h}, 55 \%), B \rightarrow(\sim 44 \mathrm{~h}, 60 \%)$ and $B_{y} \rightarrow(\sim 27 \mathrm{~h}$, $53 \%)$. For the two quantities $B$ and $B_{y}$ it may be said that the efficiency functions are more distinct if a stable sector structure for the IMF is present for several days, i.e. if the interplanetary magnetic field is structured in a CIR pattern. If the shapes of all four efficiency functions are compared to each other, one realizes that the ram pressure exerts the most significant influence on SKR at a certain lag time. Since $P_{r a m}$ is interrelated with $v, B$ and $B_{y}$ in a CIR compression region it is not surprising that also the latter yield similar results for the LPT computations. The important role of the ram pressure for the enhancement of SKR activity will be discussed below on the basis of a model proposed by Cowley et al. (2005).

- Mixing of $P_{\text {ram }}, v, B$ and $B_{y}$ in the frame of a multichannel filter does not enhance the result since included variations and lag times are too different to complement each other. The efficiency function for the multi-channel computations achieves very high values $(\sim 85 \%)$ but it does not level off at a constant plateau level before the maximum temporal shift of $57.6 \mathrm{~h}$ is reached.

- The components $B_{x}$ and $B_{z}$ of the IMF are poor quantities for explaining the external control of SKR. The efficiency functions are either low $(\leq 25 \%)$ or the filtering does not seem to be completed until the maximum lag time is reached. This does not exclude the possibility that a significant influence of $B_{x}$ or $B_{z}$ on SKR may be found at greater lag times beyond $57 \mathrm{~h}$.

- The Akasofu-parameter yields a quite good linear prediction ( $\sim 46 \mathrm{~h}$ lag time, $47 \%$ efficiency) which is, however, less significant than results found for the ram pressure. The modified Akasofu-parameter and the reconnection voltage exhibit much poorer correlations with the SKR intensity profile. It seems that the event of prominent SKR intensification occurring around DOY 233, 2004 is not a result of enhanced substorm activity initiated by an increased production of open flux in the frame of dayside magnetopause reconnection. As mentioned above, the predominantly orientation of the IMF is from north to south during several days before DOY 233 thus providing unfavorable conditions for dayside magnetopause reconnection. The maximum of the IMF magnetic field strength does not exceed $1.1 \mathrm{nT}$.

- The proton temperature and the instability quantity for the Kelvin-Helmholtz instability also yield poor results as far as the linear prediction of SKR is concerned. In the following a short comment on the profile of the KH-instability quantity $Q$ is given. One discrete value of the $Q$-profile was obtained by searching for the maximum of $Q$ along the dayside magnetopause at a discrete time. So, the obtained profile is actually a $Q_{\max }$-profile assuming that the position of $Q_{\max }$ at the dayside magnetopause can be magnetically linked to the true SKR source region near Saturn. If the magnetic field lines which are penetrating the SKR source region refer to another magnetopause position different from the $Q_{\max }$-position then another $Q$ must be considered for the profile. A more detailed study on the basis of Direction-Finding for deriving the correct longitude of the source position would be required resulting in a different profile for $Q$. Therefore, Cassini must be positioned sufficiently close to the planet because DirectionFinding has an inaccuracy for the SKR source position in the order of $1^{\circ}-2^{\circ}$ (Cecconi and Zarka, 2005a). So, taking the $Q_{\max }$-quantity directly as input for the linear prediction studies seems to be an insufficient approach in this respect.

- For the output, an intensity profile integrated over frequency seems to be the best choice. As modified output signals a mean intensity, the upper frequency limit of SKR, the lower frequency limit and the frequency bandwidth have also been tested. These modifications for the output yield lower efficiencies. Especially for the outputs concerning the frequency limits, characteristic lag times are shifted to higher values. Furthermore, it was found that the efficiency function for the integrated 
intensity profile can be raised further if the integration is just performed from $300-1200 \mathrm{kHz}$ thereby skipping intensity fluctuations which are caused by variations of the lower frequency limit. Thus, these variations seem to be rather due to an internal process, e.g. the rotation of the planet in combination with the specific beaming of SKR, than to an external influence from the solar wind. So, the integrated SKR intensity profile must be handled with care because it represents SKR activity which was beamed towards a distant observer, i.e. Cassini, located at a specific position around Saturn. This measured profile can by no means represent SKR activity for all possible source regions which are stimulated by the solar wind. Furthermore, slight changes in the longitude or latitude of the source region together with the hollow-cone-shaped beaming structure can cause huge intensity variations seen by a quasi-fixed distant observer (Cecconi and Zarka, 2005b).

According to the model of Cowley et al. (2005) intensified auroral and SKR emissions are caused by a compression of Saturn's magnetosphere as a response to an increased solar wind ram pressure in a CIR compression region. A compression of Saturn's magnetosphere causes reconnection in open tail lobes due to an enhancement of current densities in the nightside plasma sheet and associated instability configurations. As a consequence, a substantial fraction of preexisting open flux is closed $(\sim 20 \mathrm{GWb})$ during several hours leading to injection of hot plasma into the outer magnetosphere of the nightside polar cap. Newly closed flux tubes are then subcorotated with $50 \%-80 \%$ of rigid corotation from the midnight sector over the dawn sector towards noon due to ionospheric torque. This picture coincides well with HST observations of Saturn's auroral structures. About $10 \mathrm{~h}$ after the onset of the magnetospheric compression bright auroras in the dawn sector have been observed (Clarke et al., 2005). Moreover, hot plasma flows in connection with a substantial enhancement of auroral emissions have been detected by Mitchell et al. (2005) and Bunce et al. (2005). The $\sim 13 \mathrm{~h}$ lag time found for the ram pressure thus includes the time required to close a substantial amount of open flux after the onset of magnetospheric compression and to subcorotate an activated SKR source region to the dawn or noon sector from where the radiation is beamed towards the Cassini spacecraft.

\section{Appendix A}

\section{Derivation of the filter coefficients for a single-channel filter}

First, the used time series (input $X_{t}$ and measured output $Z_{t}$ ) must be centered around their respective means and they must be normalized to standard deviation units to ensure that variations and amplitude values of different time series become directly comparable. Centered and normalized time series are indicated by a $\sim$-sign, e.g. $\tilde{X}_{t}$, in order to distinguish them from the original data. The error between the measured output $\tilde{Z}_{t}$ and the calculated output $\tilde{Y}_{t}$ is simply defined as

$e_{t}=\tilde{Z}_{t}-\tilde{Y}_{t}=\tilde{Z}_{t}-\sum_{s=-m}^{M-1} f_{s} \tilde{X}_{t-s}$.

$\tilde{Y}_{t}$ is replaced by the filtered input signal as outlined in Eq. (1). The mean-square-error, i.e. the error power $I$, has to be minimized. If a time series consists of $N$ discrete values then $I$ is given as

$$
\begin{aligned}
I & =\frac{1}{N-1} \sum_{t=0}^{N-1} e_{t}^{2} \\
& =\frac{1}{N-1} \sum_{t=0}^{N-1}\left[\tilde{Z}_{t}-\sum_{s=-m}^{M-1} f_{s} \tilde{X}_{t-s}\right]^{2} \rightarrow \text { minimize }
\end{aligned}
$$

Minimizing means that the derivation of $I$ after the filter coefficients $f_{s}$ must be set equal to zero. A total of $(M+m)$ filter coefficients results in $(M+m)$ derivations and hence in a system of $(M+m)$ normal equations according to

$$
\begin{aligned}
& \frac{1}{N-1} \sum_{t=0}^{N-1} \tilde{Z}_{t} \tilde{X}_{t-v}- \\
& \sum_{s=-m}^{M-1} f_{s} \frac{1}{N-1} \sum_{t=0}^{N-1} \tilde{X}_{t-s} \tilde{X}_{t-v}=0 \\
& \quad \text { with } v=-m, \ldots, M-1
\end{aligned}
$$

The index $v$ indicates the index $s$ for which the derivation is currently performed. One recognizes that the first sum over $t$ is the definition of the cross-correlation coefficient between $Z_{t}$ and $X_{t}$ for a certain temporal shift $v$. All $(M+m)$ crosscorrelation coefficients as a function of the temporal shift parameter $v$ can be summarized in a vector named $\boldsymbol{G}_{\boldsymbol{v}}$. Similarly, the second sum over $t$ in Eq. (A4) represents a vector $\boldsymbol{R}_{\boldsymbol{v}}$ storing all $(M+m)$ auto-correlation coefficients of $X_{t}$ for a constant $s$. If the sum over $s$ is incorporated, the second term on the left side of Eq. (A4) can be written in matrix notation as

$$
\begin{gathered}
\sum_{s=-m}^{M-1} f_{s} \frac{1}{N-1} \sum_{t=0}^{N-1} \tilde{X}_{t-s} \tilde{X}_{t-v}= \\
=\sum_{s=-m}^{M-1} f_{s} \boldsymbol{R}_{\boldsymbol{v}, s}=\mathbf{R}_{\mathbf{v s}} f_{s} .
\end{gathered}
$$

$\mathbf{R}_{\mathbf{v s}}$ becomes a square matrix storing all auto-correlation coefficients of $X_{t}$ as a function of the shift parameters $v$ $(v=-m, \ldots, M-1$; row index $)$ and $s(s=-m, \ldots, M-1$; column index). $\mathbf{R}_{\mathbf{v s}}$ fulfills the characteristics of a Toeplitz. matrix (Press et al., 1986) which simplifies its computation.

So, Eq. (A4) can be written in matrix notation as

$$
\mathbf{R}_{\mathrm{vs}} f_{s}=G_{v} \text {. }
$$


The solution of this matrix equation yields a vector storing the filter coefficients according to

$f_{s}=\left(\mathbf{R}_{\mathrm{vs}}\right)^{-1} \boldsymbol{G}_{\boldsymbol{v}}$.

The computation of the filter coefficients can be summarized as follows:

- calculate the cross-correlation coefficients between the measured input $X_{t}$ and the measured output $Z_{t}$ as a function of the temporal shift and arrange these coefficients in a vector $\boldsymbol{G}_{\boldsymbol{v}}$

- calculate the auto-correlation coefficients for the input $X_{t}$ as a function of the temporal shift and arrange these coefficients in a matrix $\mathbf{R}_{\mathbf{v s}}$ (Toeplitz matrix)

- insert $\boldsymbol{G}_{v}$ and $\mathbf{R}_{\mathrm{vs}}$ into Eq. (A7) and compute the filter coefficients $f_{s}$.

Acknowledgements. Topical Editor I. A. Daglis thanks P. Galopeau and another referee for their help in evaluating this paper.

\section{References}

Akasofu, S.-I.: Interplanetary energy flux associated with magnetospheric substorms, Planet. Space Sci., 27, 425-431, 1978.

Bunce, E. J., Cowley, S. W. H., Wright, D. M., Coates, A. J., Dougherty, M. K., Krupp, N., Kurth, W. S., and Rymer, A. M.: In situ observations of a solar wind compression-induced hot plasma injection in Saturn's tail, Geophys. Res. Lett., 32, L20S04, 2005.

Cecconi, B., and Zarka, P.: Direction finding and antenna calibration through analytical inversion of radio measurements performed using a system of two or three electric dipole antennas on a three-axis stabilized spacecraft, Radio Sci., 40, RS3003, doi:10.1029/2004RS003070, 2005a.

Cecconi, B. and Zarka, P.: Model of a variable radio period for Saturn, J. Geophys. Res., 110, A12203, 2005b.

Clarke, J. T., Gérard, J.-C., Grodent, D., Wannawichian, S., Gustin, J., Connerney, J., Crary, F., Dougherty, M., Kurth, W., Cowley, S. W. H., Bunce, E. J., Hill, T., and Kim, J.: Morphological differences between Saturn's ultraviolet aurorae and those of Earth and Jupiter, Nature, 433, 717-719, 2005.

Cowley, S. W. H., Badman, S. V., Bunce, E. J., Clarke, J. T., Gérard, J.-C., Grodent, D., Jackman, C. M., Milan, S. E., and Yeoman, T. K.: Reconnection in a rotation-dominated magnetosphere and its relation to Saturn's auroral dynamics, J. Geophys. Res., 110, A02201, 2005.

Crary, F. J., Clarke, J. T., Dougherty, M. K., Hanlon, P. G., Hansen, K. C., Steinberg, J. T., Barraclough, B. L., Coates, A. J., Gérard, J.-C., Grodent, D., Kurth, W. S., Mitchell, D. G., Rymer, A. M., and Young, D. T.: Solar wind dynamic pressure and electric field as the main factors controlling Saturn's aurorae, Nature, 433, 720-722, 2005.

Desch, M. D.: Evidence for solar wind control of Saturn radio emission, J. Geophys. Res., 87, 4549-4554, 1982.

Desch, M. D.: Radio emission signature of Saturn immersions in Jupiter's magnetic tail, J. Geophys. Res., 88, 6904-6910, 1983.
Desch, M. D. and Rucker, H. O.: The relationship between Saturn kilometric radiation and the solar wind, J. Geophys. Res., 88, 8999-9006, 1983.

Desch, M. D. and Rucker, H. O.: Saturn radio emission and the solar wind: Voyager-2 studies, Adv. Space Res., 5, 333-336, 1985.

Dougherty, M. K., Kellock, S., Southwood, D. J., Balogh, A., Smith, E. J., Tsurutani, B. T., Gerlach, B., Glassmeier, K.-H., Gleim, F., Russell, C. T., Erdos, G., Neubauer, F. M., and Cowley, S. W. H.: The Cassini magnetic field investigation, Space Sci. Rev., 114, 331-383, 2004.

Farrell, W. M., Desch, M. D., Kaiser, M. L., Lecacheux, A., Kurth, W. S., Gurnett, D. A., Cecconi, B., and Zarka, P.: A nightside source of Saturn's kilometric radiation: Evidence for an inner magnetosphere energy driver, Geophys. Res. Lett., 32, L18107, 2005.

Gallagher, D. L., and D'Angelo, N.: Correlations between solar wind parameters and auroral kilometric radiation intensity, Geophys. Res. Lett., 8, 1087-1089, 1981.

Galopeau, P. H. M., Zarka, P., and Le Quéau, D.: Source location of Saturn's kilometric radiation: The Kelvin-Helmholtz instability hypothesis, J. Geophys. Res., 100, 26 397-26410, 1995.

Gosling, J. T.: Corotating and transient solar wind flows in three dimensions, Annu. Rev. Astron. Astrophys., 34, 35-74, 1996.

Gurnett, D. A., Kurth, W. S., Kirchner, D. L., Hospodarsky, G. B., Averkamp, T. F., Zarka, P., Lecacheux, A., Manning, R., Roux, A., Canu, P., Cornilleau-Wehrlin, N., Galopeau, P., Meyer, A., Boström, R., Gustafsson, G., Wahlund, J.-E., Åhlen, L., Rucker, H. O., Ladreiter, H. P., Macher, W., Woolliscroft, L. J. C., Alleyne, H., Kaiser, M. L., Desch, M. D., Farrell, W. M., Harvey, C. C., Louarn, P., Kellogg, P. J., Goetz, K., and Pedersen, A.: The Cassini radio and plasma wave investigation, Space Sci. Rev., 114, 395-463, 2004.

Gurnett, D. A., Kurth, W. S., Hospodarsky, G. B., Persoon, A. M., Averkamp, T. F., Cecconi, B., Lecacheux, A., Zarka, P., Canu, P., Cornilleau-Wehrlin, N., Galopeau, P., Roux, A., Harvey, C., Louarn, P., Bostrom, R., Gustafsson, G., Wahlund, J.-E., Desch, M. D., Farrell, W. M., Kaiser, M. L., Goetz, K., Kellogg, P. J., Fischer, G., Ladreiter, H.-P., Rucker, H., Alleyne, H., and Pedersen, A.: Radio and plasma wave observations at Saturn from Cassini's approach and first orbit, Science, 307, 12551259, 2005.

Jackman, C. M., Achilleos, N., Bunce, E. J., Cowley, S. W. H., Dougherty, M. K., Jones, G. H., Milan, S. E., and Smith, E. J.: Interplanetary magnetic field at $\sim 9 \mathrm{AU}$ during the declining phase of the solar cycle and its implications for Saturn's magnetospheric dynamics, J. Geophys. Res., 109, A11203, 2004.

Kaiser, M. L., Desch, M. D., Warwick, J. W., and Pearce, J. B.: Voyager detection of nonthermal radio emission from Saturn, Science, 209, 1238-1240, 1980.

Kaiser, M. L., Desch, M. D., Kurth, W. S., Lecacheux, A., Genova, F., Pedersen, B. M., and Evans, D. R.: Saturn as a radio source, in: Saturn, edited by: Gehrels, T. and Matthews, M. S., Univ. of Arizona Press, Tucson, 378-415, 1984.

Kurth, W. S., Gurnett, D. A., Clarke, J. T., Zarka, P., Desch, M. D., Kaiser, M. L., Cecconi, B., Lecacheux, A., Farrell, W. M., Galopeau, P., Gérard, J.-C., Grodent, D., Prangé, R., Dougherty, M. K., and Crary, F. J.: An Earth-like correspondence between Saturn's auroral features and radio emission, Nature, 433, 722$725,2005 a$. 
Kurth, W. S., Hospodarsky, G. B., Gurnett, D. A., Cecconi, B., Louarn, P., Lecacheux, A., Zarka, P., Rucker, H. O., Boudjada, M., and Kaiser, M. L.: High spectral and temporal resolution observations of Saturn kilometric radiation, Geophys. Res. Lett., 32, L20S07, 2005b.

Lecacheux, A., Galopeau, P., and Aubier, M.: Re-visiting Saturnian kilometric radiation with Ulysses/URAP, in: Planetary Radio Emissions IV, edited by Rucker, H. O., Bauer, S. J. and Lecacheux, A., Austrian Academy of Sciences Press, Vienna, 313-325, 1997.

Levinson, N.: The Wiener RMS (root-mean-square) error criterion in filter design and prediction, Appendix B, in: Extrapolation, interpolation, and smoothing of stationary time series with engineering applications, edited by: Wiener, N., 1949.

Mitchell, D. G., Brandt, P. C., Roelof, E. C., Dandouras, J., Krimigis, S. M., Mauk, B. H., Paranicas, C. P., Krupp, N., Hamilton, D. C., Kurth, W. S., Zarka, P., Dougherty, M. K., Bunce, E. J., and Shemansky, D. E.: Energetic ion acceleration in Saturn's magnetotail: substorms at Saturn?, Geophys. Res. Lett., 32, L20S01, 2005.

Press, W. H., Flannery, B. P., Teukolsky, S. A., and Vetterling, W. T.: Numerical Recipes, The art of scientific computing, Cambridge University Press, 1986.

Schönwiese, C.-D.: Praktische Statistik für Meteorologen und Geowissenschafter, Geb. Bornträger, Berlin-Stuttgart, 1985.

Slavin, J. A., Smith, E. J., Spreiter, J. R., and Stahara, S. S.: Solar wind flow about the outer planets: gas dynamic modeling of the Jupiter and Saturn bow shocks, J. Geophys. Res., 90, 6275-6286, 1985.

Taubenheim, J.: Statistische Auswertung geophysikalischer und meteorologischer Daten, Verlagges. Geest \& Porting, Leipzig, 1969.

Trauger, J. T., Griffiths, R. E., Hester, J. J., Hoessel, J. G., Holtzman, J. A., Krist, J. E., Mould, J. R., Sahai, R., Scowen, P. A., Stapelfeldt, K. R., and Watson, A. M.: Saturn's hydrogen aurora: wide-field planetary camera 2 imaging from the Hubble Space Telescope, J. Geophys. Res., 103, 20 237-20 244, 1998.
Tsurutani, B. T., Smith, E. J., Pyle, K. R., and Simpson, J. A.: Energetic protons accelerated at corotating shocks - Pioneer 10 and 11 observations from 1 to $6 \mathrm{AU}$, J. Geophys. Res., 87, 73897404, 1982.

Vasyliunas, V. M., Kan, J. R., Siscoe, G. L., and Akasofu, S.I.: Scaling relations governing magnetosphere energy transfer, Planet. Space Sci., 30, 359-365, 1982.

Warwick, J. W., Pearce, J. B., Evans, D. R., Carr, T. D., Schauble, J. J., Alexander, J. K., Kaiser, M. L., Desch, M. D., Pedersen, B. M., Lecacheux, A., Daigne, G., Boischot, A., and Barrow, C. H.: Planetary radio astronomy observations from Voyager 1 near Saturn, Science, 215, 239-243, 1981.

Warwick, J. W., Evans, D. R., Romig, J. H., Alexander, J. K., Desch, M. D., Kaiser, M. L., Aubier, M., Leblanc, Y., Lecacheux, A., and Pedersen, B. M.: Planetary radio astronomy observations from Voyager 2 near Saturn, Science, 215, 582-587, 1982.

Wiener, N.: Extrapolation, interpolation, and smoothing of stationary time series with engineering applications, Publ. Techn. Press of the M.I.T. and John Wiley \& Sons Inc., New York, 1949.

Wu, C. S., and Lee, L. C.: A theory of terrestrial kilometric radiation, Astrophys. J., 230, 621-626, 1979.

Young, D. T., Berthelier, J. J., Blanc, M., Burch, J. L., Coates, A. J., Goldstein, R., Grande, M., Hill, T. W., Johnson, R. E., Kelha, V., McComas, D. J., Sittler, E. C., Svenes, K. R., Szegö, K., Tanskanen, P., Ahola, K., Anderson, D., Bakshi, S., Baragiola, R. A., Barraclough, B. L., Black, R. K., Bolton, S., Booker, T., Bowman, R., Casey, P., Crary, F. J., Delapp, D., Dirks, G., Eaker, N., Funsten, H., Furman, J. D., Gosling, J. T., Hannula, H., Holmlund, C., Huomo, H., Illiano, J. M., Jensen, P., Johnson, M. A., Linder, D. R., Luntama, T., Maurice, S., McCabe, K. P., Mursula, K., Narheim, B. T., Nordholt, J. E., Preece, A., Rudzki, J., Ruitberg, A., Smith, K., Szalai, S., Thomsen, M. F., Viherkanto, K., Vilppola, J., Vollmer, T., Wahl, T. E., West, M., Ylikorpi, T., and Zinsmeyer, C.: Cassini Plasma Spectrometer Investigation, Space Sci. Rev., 114, 1-112, 2004. 